\title{
A Secure Peer-to-Peer Group Collaboration Scheme for Healthcare System*
}

\author{
Byong-In Lim, Kee-Hyun Choi, and Dong-Ryeol Shin \\ School of Information and Communication Engineering, \\ Sungkyunkwan University, \\ 440-746, Suwon, Korea, +82-31-290-7125 \\ \{1bi77, gyunee, drshin\}@ece.skku.ac.kr
}

\begin{abstract}
P2P (Peer-to-Peer) applications allow flexible organization, distribution of role to participating peers and the ability to share information with the other peers for group collaborations. As a result, P2P systems are not only gaining importance, but also becoming ubiquitous media for information exchange. JXTA is a P2P application development infrastructure that enables developers to easily create service-oriented software. This paper presents a lowcost, patient-friendly JXTA-based healthcare system, which is comprised of medical sensor modules in conjunction with wireless communication technology. Among the most important activities associated with the healthcare system are the sharing of medical information and collaborative medical work. The JXTA grouping service facilitates to share data in a secure manner under P2P environments. Through JXTA grouping service, we implemented a prototyped system, which improves the ability of the healthcare workers to cope with dynamic situation, which in turn makes it possible to offer more efficient medical services.
\end{abstract}

\section{Introduction}

Advancements in sensor, information and communication technology can play an important role in achieving cost reduction and efficiency improvement in healthcare delivery systems, to the extent that this offers high-quality medical service anytime and anywhere. However, conventional healthcare systems, including the Mobihealth [1] project, utilize a central server for the look up of information. Furthermore, the possibility of offering seamless service is limited by the network connectivity of wireless devices or resource-constraints. In addition, traditional systems only focus on organic communication and service utilization between patients and hospital, whereas they ignore the systematic communication and sharing of information between healthcare workers in the hospital. For these reasons, these systems cannot cope with acute situations dynamically and promptly. Also, privacy and security are potential problems. Patient's data should be available irrespective of their location, but only to authorized healthcare workers.

* This research was partially supported by a grant from the CUCN, Korea and Korea Science \& Engineering Foundation (R01-2004-000-10755-0) 
To resolve these problems, we propose a JXTA-based healthcare system, which operates in a peer-to-peer (P2P) environment so as to offer seamless service by distributing the healthcare services among the healthcare workers. This sharing of information about the medical treatment that patients receive between the healthcare workers in the JXTA environment improves their ability to cope with dynamic situations, which in turn makes it possible to offer more efficient medical services. Furthermore, the JXTA grouping service makes it possible to share data in a secure manner under the $\mathrm{P} 2 \mathrm{P}$ environment. So, our system protects patient's data. In this paper, we focus on the system architecture design in hospital region which is based on JXTA grouping mechanisms.

This paper is structured as follows: Section 2 discusses system architecture for healthcare services and the system implementation is presented in section 3. Finally, this paper is concluded in section 4 .

\section{System Architecture for Healthcare Services}

\subsection{Architecture Overview}

Our system consists of two regions, viz. the Healthcare region and the Hospital region, as shown in Figure 1.

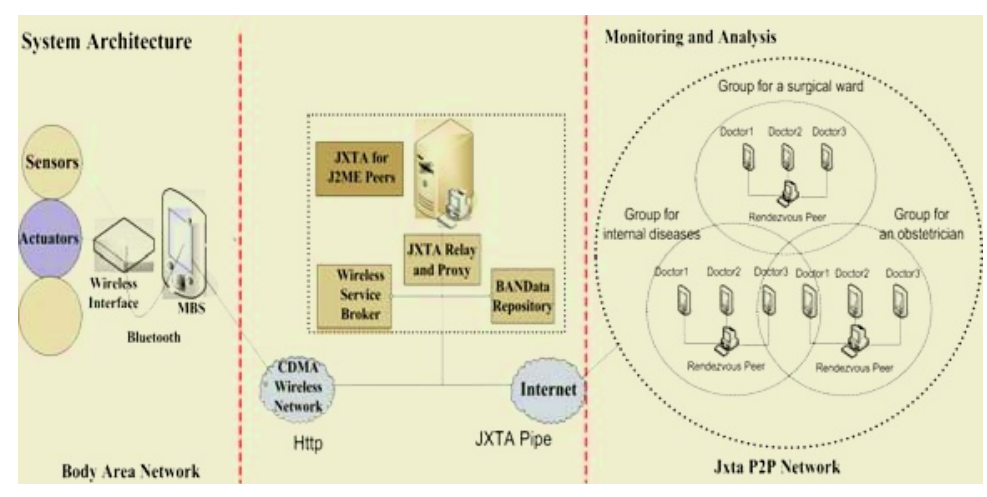

Fig. 1. System Architecture

The healthcare BAN consists of sensors, a Wireless Interface, PDA communication, and various facilities. The Hospital region is composed of the JXTA P2P network that supports the doctor's mobility and dynamic service management modules. A P2P network distributes the information among the member nodes, instead of concentrating it at a single server. A JXTA relay peer is defined among the two regions, in order to provide a systematic computing environment between the Healthcare region and the Hospital region. JXTA relays act as proxies for the individual patients' PDAs, as well as taking care of all the heavier tasks on their behalf. For more details, refer to [2]. In next section, we describe how healthcare services with collaboration of JXTA secure group environment are formed and managed in a hospital region. 


\subsection{Secure Group Collaboration Scheme in Hospital}

Among the most time consuming activities associated with the public health sector are documentation and data exchange. In a hospital, the healthcare workers form a peer group within the institution. Together they can create a secure peer group with various access rights, so as to allow the sharing of the patient's data (e.g. his or her patient UUID, patient sensor data, etc) and other kind of information (e.g. a first-aid, drugs to prescribe, etc) under the secure environment. Figure 2 shows a sequence diagram of group collaboration scheme in hospital domains.

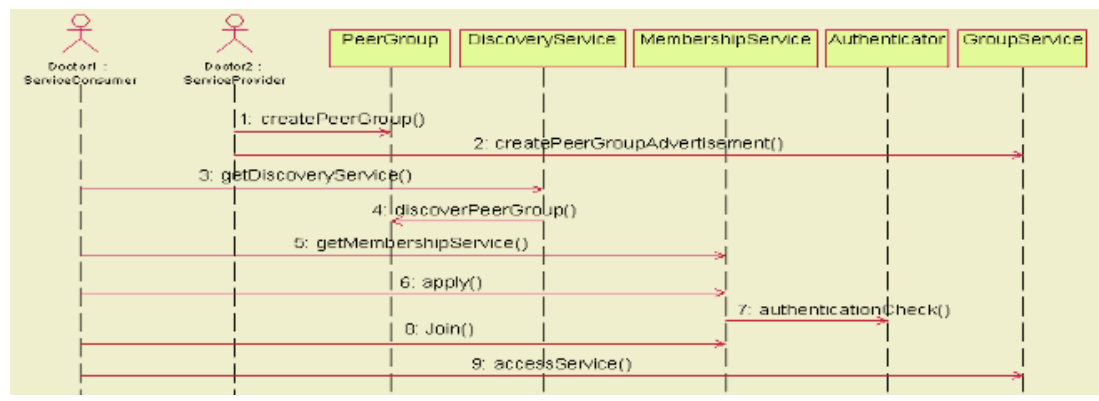

Fig. 2. Sequence Diagram of Group Collaboration Scheme

JXTA combines the overlay network with a namespace abstraction referred to as a peer group [3]. Any peer may create a new peer group at any time for any reason to communicate with any set of peers. The only requirement is that all peers have agreed upon a common set of services and protocols for implementing secure group collaboration (authentication, authorization, message propagation, discovery service, etc). Consequently, JXTA grouping service is an effective means to implement P2P collaboration system under secure environment. This is the main reason for which the healthcare system is based on the JXTA platform. In our system, the healthcare region consists of several groups (e.g. groups corresponding to specific surgical wards, dental treatment, etc). The collaboration scheme of secure peer group is as follows. Before healthcare workers can interact with the group, they need to create a peer group. When creating the peer group, the healthcare workers involved initialize the local secure environment, and then publish a peer group advertisement, which includes the peer group ID and peer group medical service. After the secure group is created, they can perform collaborative service and functionality of the peer. Searching and sharing are done on the peer group level, i.e., shared contents are only available to the peer group. In order for service consumers to utilize medical service, they must firstly achieve their guaranteed passwords as well as the information of secure peer groups which provide medical service in the JXTA network. In JXTA, the membership service is used to apply for peer group membership, join a peer group, and exit from a peer group. The membership service allows a peer to establish an identity within a peer group. Once an identity has been established, a credential is available, which allows the peer to prove that they have obtained the identities rightfully. In our system, the security is ensured by using JXTA grouping mechanism to implement group level security policies for 
membership and service access. Also, the JXTA grouping mechanism supports the sharing of medical information and collaborative medical work, which in turn makes it possible to offer more efficient medical services.

\section{Implementation}

In this study, we utilize the JXTA Platform to develop a medical-service management tool as well as secure group collaborations in a hospital domain. We also implemented a software for monitoring the patient's status, in the BAN as well as in

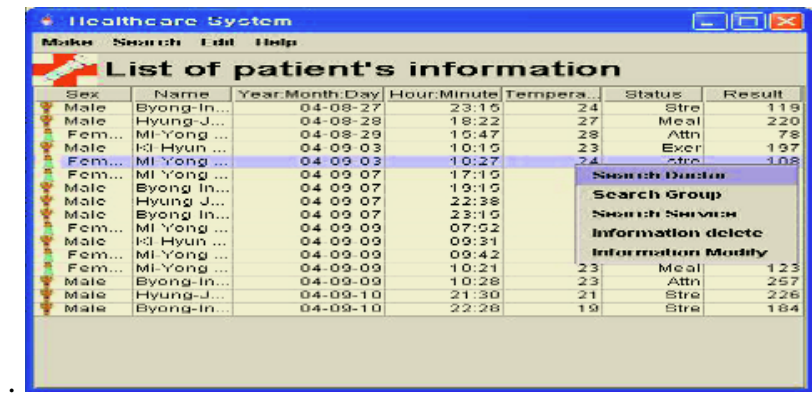

Fig. 3. Data Management JXTA Application

the JXTA application used for managing the patient's information; medical status and personal information, as shown in Figure 3. The use of the JXTA Platform with its discovery and grouping mechanisms enables us to offer efficient and adaptive medical services.

\section{Conclusion}

In this paper we have presented the design of a JXTA-based healthcare system which enables healthcare workers to share data in a secure manner under the P2P environment. This paradigm offers significant advantages in healthcare workers to cope with dynamic situations, thus improving the quality of the medical service that they can provide. Future work includes the detailed simulations and performance evaluations for the developed systems.

\section{References}

1. Nikolay Dokovsky, Aart van Halteren, Ing Widya, "BANip: enabling remote healthcare monitoring with Body Area Networks", International Workshop on scientific engineering of Distributed Java applications, November 27-28, 2003.

2. Byongin Lim, et al, "A JXTA-based Architecture for Efficient and Adaptive Healthcare Services”, ICOIN 2005, LNCS 3391, pp. 776-785, January 31- February 2, 2005.

3. PKI Security for JXTA Overlay Network, Jeff Altman, February 2003. 